\title{
Expressions of miR-122a and miR-3195 \\ in laryngeal cancer and their effects on the proliferation and apoptosis of laryngeal cancer cell Hep-2
}

\author{
Xingli Jiang ${ }^{1, A-F}$, Zhiguang Gao ${ }^{2, A-C}$, Linli Tian ${ }^{1, D, F}$, Ming Liu, ${ }^{1, A, E, F}$ \\ ${ }^{1}$ Department of Otorhinolaryngology, and Head and Neck Surgery, The Second Affiliated Hospital of Harbin Medical University, China \\ ${ }^{2}$ Department of Otorhinolaryngology, and Head and Neck Surgery, Heilongjiang Provincial Hospital Affiliated to Harbin Institute of Technology, China \\ A - research concept and design; $B$ - collection and/or assembly of data; $C$ - data analysis and interpretation; \\ $D$ - writing the article; $E$ - critical revision of the article; $F$ - final approval of the article
}

\section{Address for correspondence \\ Ming Liu}

E-mail: liuming83b@163.com

Funding sources

None declared

\section{Conflict of interest}

None declared

Received on August 9, 2019

Reviewed on November 13, 2019

Accepted on March 10, 2020

Published online on May 28, 2020

Cite as

Jiang X, Gao Z, Tian L, Liu M. Expressions of miR-122a and miR-3195 in laryngeal cancer and their effects on the proliferation and apoptosis of laryngeal cancer cell Hep-2. Adv Clin Exp Med. 2020;29(5):525-534.

doi:10.17219/acem/118848

DOI

10.17219/acem/118848

Copyright

Copyright by Author(s)

This is an article distributed under the terms of the

Creative Commons Attribution 3.0 Unported (CC BY 3.0)

(https://creativecommons.org/licenses/by/3.0/)

\begin{abstract}
Background. Laryngeal cancer (LC) is one of the common malignant tumors in the head and neck area, and the survival rate for patients is low.

Objectives. To investigate miR-122a and miR-3195 expressions in LC tissue, their correlations with clinicopathological features, and their impacts on Hep-2 proliferation and apoptosis.

Material and methods. Thirty $L C$ and 20 peritumoral tissue specimens were analyzed. miR-122a, miR-122a-negative control sequence, miR-3195, and miR-3195-NG sequence were transfected into Hep-2 in the miR-122a-mimics, miR-122a-NG, miR-3195-mimics, and miR-3195-NG groups, respectively. The miR-122a-mimics-non-transfected and miR-3195-mimics-non-transfected groups used non-transfected Нер-2.

Results. There were lower miR-122a, miR-3195 and occludin protein, and higher TBX1 protein expressions in LC than in the peritumoral tissue; the miR-122a level was associated with clinical stage (all $p<0.001$ ). Positive correlations between miR-122a and miR-3195, and miR-122a and occludin expressions, and a negative correlation between miR-3195 and TBX1 expressions were observed $(r=0.418, r=0.541, r=-0.428$, all $p<0.001$ ). The miR-122a and miR-3195 levels in the 2 mimics groups increased respectively compared to their $N G$ and the non-transfected groups. At different time points after $24 \mathrm{~h}$ of transfection, the optical density in the 2 mimics groups was lower than in their NG groups. The miR-122a-mimics group had an increased occludin level and the miR-3195-mimics group had a decreased TBX1 level, and both groups had greater apoptosis rates than their NG groups and in the non-transfected groups (all p <0.001).
\end{abstract}

Conclusions. miR-122a is associated with clinical stage. miR-122a and miR-3195 may act as tumor suppressors and play a role in LC pathogenesis. They can suppress Hep-2 proliferation and promote its apoptosis, probably owing to the upregulation of occludin by miR-122a and suppression of TBX1 by miR-3195.

Key words: apoptosis, microRNAs, cell proliferation, laryngeal neoplasms 


\section{Introduction}

Laryngeal cancer (LC) is one of the common malignant tumors in the head and neck area, which accounts for 85-90\% of the malignant tumors in the larynx; currently, surgery, chemotherapy and radiotherapy are the main methods for treating LC. ${ }^{1}$ Although technologies in cancer treatment have been advancing over the past few decades, many patients still experience metastasis of tumor cells into important organs, leading to low survival rates. ${ }^{2}$ This problem is mainly caused by a lack of early diagnosis and leads to a decrease in treatment efficacy; apart from that, patients' weak tolerance to chemotherapy or radiotherapy and postoperative recurrence and metastasis of tumor also contribute to the high mortality. ${ }^{3}$ Therefore, it is of great significance to find molecular biomarkers that are related to the early diagnosis of $\mathrm{LC}$, the molecular mechanisms in LC pathogenesis, and the biological marker and therapeutic target that can inhibit LC occurrence and progression in order to improve early diagnosis and prognosis in patients.

The microRNA (miRNA), existing widely in eukaryotes, is a highly conserved endogenous non-coding hairpinshaped nucleotide transcript (containing 19-25 base and 18-25 nucleotides). ${ }^{4,5}$ Some studies have found that miRNA is closely associated with LC occurrence and progression. Liu et al. have reported that miR-125a can target hematopoietic stem cell-specific protein 1-associated protein X-1 (HAX-1) and reverse the cisplatin resistance in Hep-2 cancer stem cell. ${ }^{6}$ Although miR-122a has been reported by Chen et al. to be able to suppress the proliferation of LC cell Hep-2, the effect of miR-122a on Hep-2 apoptosis remains unclear. ${ }^{7}$ Previous studies have demonstrated that miR-3195 can act as a tumor suppressor gene. For instance, Yoon et al. reported a reduction in miR-3195 expression level in gastric cancer tissue. ${ }^{8}$ However, there have yet been no studies on the expression of miR-3195 in LC.

Thus, in the present study, we measured the expressions of miR-122a and miR-3195 in LC tissue and Hep-2 cell and investigated these 2 miRNAs in LC pathogenesis and their impacts on the cell proliferation and apoptosis, with the hope of gaining a better understanding on the biological function of these 2 miRNAs in LC. Furthermore, as a preliminary analysis of the molecular mechanisms of miR-122a and miR-3195, bioinformatics was also used to examine the potential target genes that may be regulated by these miRNAs.

\section{Material and methods}

\section{Basic information}

A total of $30 \mathrm{LC}$ specimens from patients undergoing surgical excision in our hospital and 20 peritumoral tissue specimens ( $>2 \mathrm{~cm}$ from the tumor) were collected for the study ( 21 men, 9 women, age range: $46-80$ years, mean age: $62.7 \pm 8.3$ years). The study was approved by the Ethics Committee of our hospital, and informed consent was obtained from all patients or their family members.

Inclusion criteria: the specimens of $\mathrm{LC}$ and peritumoral tissues were confirmed by pathological examination ${ }^{9}$; patients or their family members signed the informed consent.

Exclusion criteria: chemotherapy, radiotherapy or immunotherapy received in the past; severe liver and kidney dysfunction, connective tissue diseases, endocrine and metabolic diseases, nervous system disease, hematopoietic disorder, immune disease, and other tumors; or a history or a family history of mental illness.

We used TargetScan Release v. 7.2 online software (http://www.targetscan.org) to predict the target gene of miR-122a and miR-3195 and found that miR-122a can regulate occludin and miR-3195 can regulate $T B X 1$.

\section{Main instruments and reagents}

Main instruments and reagents were as follows: quantitative fluorescence polymerase chain reaction (PCR) (7500; Applied Biosystems, Foster City, USA), total RNA extraction kit (EasyPure miRNA Kit; Transgen, Beijing, China). PCR-reverse transcription kit (TransScript ${ }^{\circledR}$ Green miRNA Two-Step qRT-PCR SuperMix; TransGen Biotech, Beijing, China), Lipofectamine 2000 and Annexin V-FITC cell apoptosis kit (Invitrogen, Carlsbad, USA), incubator (RPMI-1640; Hyclone, Logan, USA), rabbit antihuman TBX1 primary antibody (Abcam, Cambridge, UK), rabbit anti-human occludin polyclonal antibody (Santa Cruz Biotechnology, Santa Cruz, USA), mouse antihuman $\beta$-catenin primary antibody (Boster Biological Technology, Wuhan, China), miR-122a mimics, miR-122a NG, miR-3195 mimics, and miR-3195 NG (Sangon Biotech, Shanghai, China), horseradish peroxidase (HRP)conjugated goat anti-mouse secondary antibody, MTT cell proliferation kit (Beyotime Biotechnology, Shanghai, China), FACSCanto flow cytometer (Becton Dickinson, Franklin Lakes, USA), and a microplate reader (Elx-800; BioTek, Winooski, USA). Primers of miR-122a, miR-3195 and U6 were designed and synthesized by Shanghai GenePharma Co., Ltd (Shanghai, China) (Table 1).

\section{Cell culture}

Hep-2 cells were seeded in a culture plate followed by the addition of RPMI-1640 medium containing 15\% fetal bovine serum (FBS) and 1\% mycillin/streptomycin. ${ }^{10}$ The cells were incubated in an incubator under saturated humidity and constant temperature $\left(37^{\circ} \mathrm{C}\right.$, $5 \% \mathrm{CO}_{2}$ ). The medium was changed in a timely manner, and the cells were digested with trypsin for subculture. The cells in logarithmic growth phase were collected for subsequent experiments. 
Table 1. Primer sequence

\begin{tabular}{l|c|c}
\multicolumn{1}{c|}{ Primers } & Forward primer & Reverse primer \\
\hline miR-122a & 5'-CCTTTGTGTAACTGTACGGCC-3' & 5'-CTTTGGCAGTAAATAGCTGATTCGAC-3' \\
\hline miR-3195 & 5'-AACACGGCTCACGCTTAC-3' & 5'-CCAGACCCTCAGACTTGC-3' $^{\text {U6 }}$ \\
\hline
\end{tabular}

\section{Cell transfection}

Cells in logarithmic growth phase were seeded in a 6 -well plate for culture $\left(1^{*} 10^{5} /\right.$ well). When fusion reached $60-70 \%$, transfection was performed according to the manufacturer's instructions for Lipofectamine 2000. Eighty nmol/L of miR-122a, miR-122a NG sequence, miR-3195, and miR-3195 NG sequence were transfected into Hep-2 cell in the miR-122a-mimics, miR-122a-NG, miR-3195-mimics, and miR-3195-NG groups, respectively, followed by the cell culture for $24 \mathrm{~h}$. Meanwhile, 2 nontransfected groups, miR-122a-mimics-non-transfected and miR-3195-mimics-non-transfected groups with nontransfected Hep-2 cells were set up and cultured for $24 \mathrm{~h}$. Subsequently, the medium was replaced with $50 \mathrm{ng} / \mathrm{mL}$ macrophage colony-stimulating factors for all cells (twice per day).

\section{qRT-PCR}

Total RNA extraction from all the tissues and cells was performed using EasyPure miRNA kit (TransGen Biotech). The purity, concentration and integrity of RNA were checked with a UV spectrophotometer and agarose gel electrophoresis, and the total RNAs were reversely transcribed using TransScript ${ }^{\circledR}$ miRNA RT Enzyme Mix and $2 \times$ TS miRNA Reaction Mix (TransGen Biotech) according to the manufacturer's instructions. Next, PCR was conducted for amplification, and the reaction system included $1 \mu \mathrm{L}$ of $\mathrm{cDNA}, 0.4 \mu \mathrm{L}$ of forward primer, $0.4 \mu \mathrm{L}$ of reverse primer, $10 \mu \mathrm{L}$ of $2 \times$ TransTaq $^{\circledR}$ Tip Green qPCR SuperMix, $0.4 \mu \mathrm{L}$ of $50 \times$ passive reference dye, and distilled deionized water that made the whole volume into $20 \mu \mathrm{L}$. The PCR running parameters were set as follows: $94^{\circ} \mathrm{C}$ for $30 \mathrm{~s}$ (pre-denaturation), $94^{\circ} \mathrm{C}$ for $5 \mathrm{~s}$ (denaturation) and $60^{\circ} \mathrm{C}$ for $30 \mathrm{~s}$ (annealing and extension) for 40 cycles. Each sample was set in triplicate, and the experiment was repeated 3 times. U6 was used as an internal control. Data was calculated using the $2^{-\Delta \Delta C t}$ method.

\section{Western blot}

The total proteins were isolated from the tissues and cells using radioimmunoprecipitation assay lysis buffer. The protein concentration was measured with bicinchoninic acid method, and the concentration was adjusted to $4 \mu \mathrm{g} / \mu \mathrm{L}$. After running $12 \%$ SDS-PAGE for separation, samples were transferred to polyvinylidene fluoride
(PVDF) membranes and stained with Ponceau solution followed by washing in phosphate-buffered saline/Tween (PBST) for 5 min and blocking in 5\% skim milk for $2 \mathrm{~h}$. Next, samples were incubated with primary antibodies overnight at $4^{\circ} \mathrm{C}(1: 1,000)$. The membrane was then washed to remove the primary antibodies, and the samples were incubated with HRP-conjugated goat anti-mouse secondary antibody $(1: 5,000)$ for $1 \mathrm{~h}$ at $37^{\circ} \mathrm{C}$ followed by 3 washes in Tris-buffered saline/Tween; 5 min per wash). Afterward, the membrane was placed in a darkroom for developing. Extra fluid on the membrane was dried using filter papers, and the samples were treated with enhanced chemiluminescence (ECL) for imaging. Protein band was scanned and analyzed with Gel-Pro Analyzer v. 4.0 software (Molecular Devices Corp, Bay Area, USA) to measure the grayscale. The relative protein expression was calculated as the grayscale value of the target protein band/grayscale value of the $\beta$-catenin protein band.

\section{Cell proliferation}

Cell suspension $\left(1 * 10^{5}\right)$ was prepared with cells in a logarithmic growth phase. After adjusting the cell density to $2 * 10^{4} \mathrm{~mL}$, the cells were seeded in a 96-well plate (2*103/well). The MTT assay was performed every $24 \mathrm{~h}$ 5 times to measure the cell viability (the final test was done at $120 \mathrm{~h})$. During the test, MTT solution $(10 \mu \mathrm{L}, 5 \mathrm{mg} / \mathrm{mL})$ was added into each well followed by $4 \mathrm{~h}$ of incubation. Next, formazan dissolution $(150 \mu \mathrm{L})$ was added into each well, and the plate was agitated under room temperature for $10 \mathrm{~min}$. The crystal was dissolved completely as observed under a light microscope. The optical density (OD) value at $570 \mathrm{~nm}$ was measured using a microplate reader, and the measurement was repeated 3 times for each well.

\section{Cell apoptosis}

Forty-eight hours after transfection, the cells were digested using trypsin, and cold PBS $(0.01 \mathrm{~mol} / \mathrm{L})$ was used to wash the collected cells. After centrifugation $(111.8 \times \mathrm{g}$, $25^{\circ} \mathrm{C}$ ), the supernatant was discarded, and the cells were resuspended with $100 \mu \mathrm{L} 1 \times$ binding buffer. Next, the samples were transferred to flow cytometry tubes, and $5 \mu \mathrm{L}$ 7AAD and $5 \mu \mathrm{L}$ PE annexin $V$ was added to each tube for a 15 min incubation away from light under room temperature before the addition of $400 \mu \mathrm{L} 1 \times$ binding buffer. The flow cytometry was completed within $1 \mathrm{~h}$. Each sample was measured 3 times. 


\section{Outcome measures}

Main outcome measures included protein expressions of occludin and TBX1 in LC Hep-2 after transfection and impacts of miR-122a and miR-3195 expressions in LC tissue and Hep-2 on cell proliferation and apoptosis.

Secondary outcome measures were associations of the miR-122a and miR-3195 expressions in LC tissue with clinicopathological features as well as the occludin and TBX1 protein expressions in LC tissue.

\section{Statistical analysis}

Statistical software SPSS v. 19.0 (IBM Corp., Armonk, USA) was applied for data analysis. Graphs were plotted using GraphPad Prism v. 7 (GraphPad Software Inc., San Diego, USA). Measurement data is presented as mean \pm standard deviation (SD). Comparisons between groups were conducted using independent samples t-test, while comparisons of mean values across multiple groups were performed with one-way analysis of variance (ANOVA) followed by Dunnett's t-test for two-group comparison. Pearson test was used to assess the correlation. A value of $\mathrm{p}<0.05$ was considered to indicate a statistically significant difference.

\section{Results}

\section{miR-122a and miR-3195 expressions in LC and peritumoral tissues and their correlations}

The qRT-PCR results showed that expression levels of miR-122a and miR-3195 in LC tissue were lower than those in peritumoral tissue (both $\mathrm{p}<0.001$ ). Pearson analysis found that miR-122a and miR-3195 expressions were positively associated $(\mathrm{r}=0.418, \mathrm{p}<0.001$; Table 2 , Fig. 1).
Table 2. miR-122a and miR-3195 expressions in LC and peritumoral tissues $(\mathrm{x} \pm \mathrm{SD})$

\begin{tabular}{|l|c|c|c|}
\multicolumn{1}{|c|}{ Group } & $\mathrm{n}$ & miR-122a & miR-3195 \\
\hline LC tissue & 30 & $0.731 \pm 0.117$ & $0.831 \pm 0.201$ \\
\hline Peritumoral issue & 20 & $1.187 \pm 0.315$ & $1.135 \pm 0.304$ \\
\hline $\mathrm{t}$ & & 7.244 & 4.264 \\
\hline $\mathrm{p}$ & & $<0.001$ & $<0.001$ \\
\hline
\end{tabular}

LC - laryngeal cancer.

\section{Associations of miR-122a and miR-3195 expressions with clinicopathological features}

The study results exhibited that the expression level of miR-122a in LC tissue was associated with patients' clinical stage $(\mathrm{p}<0.001)$ but not associated with patients' gender, age, pathological grade, and lymphatic metastasis (all p > 0.05). Meanwhile, the expression level of miR-3195 in LC tissue was not associated with all of these factors (all p > 0.05; Table 3).

\section{Correlation between occludin protein expression in LC tissue and miR-122a}

The occludin protein expression level in the LC issue was much lower than in peritumoral tissue $(\mathrm{p}<0.001)$. Pearson analysis showed that miR-122a expression level was positively associated with occludin protein expression level ( $\mathrm{r}=0.541, \mathrm{p}<0.001$; Fig. 2).

\section{Correlation between TBX1 protein expression in LC tissue and miR-3195}

It was found that TBX1 protein expression level in LC tissue was much higher than in peritumoral tissue $(\mathrm{p}<0.001)$. Pearson analysis showed that miR-3195 expression level in LC tissue was negatively correlated with TBX1 protein $(\mathrm{r}=-0.428, \mathrm{p}<0.001$; Fig. 3).
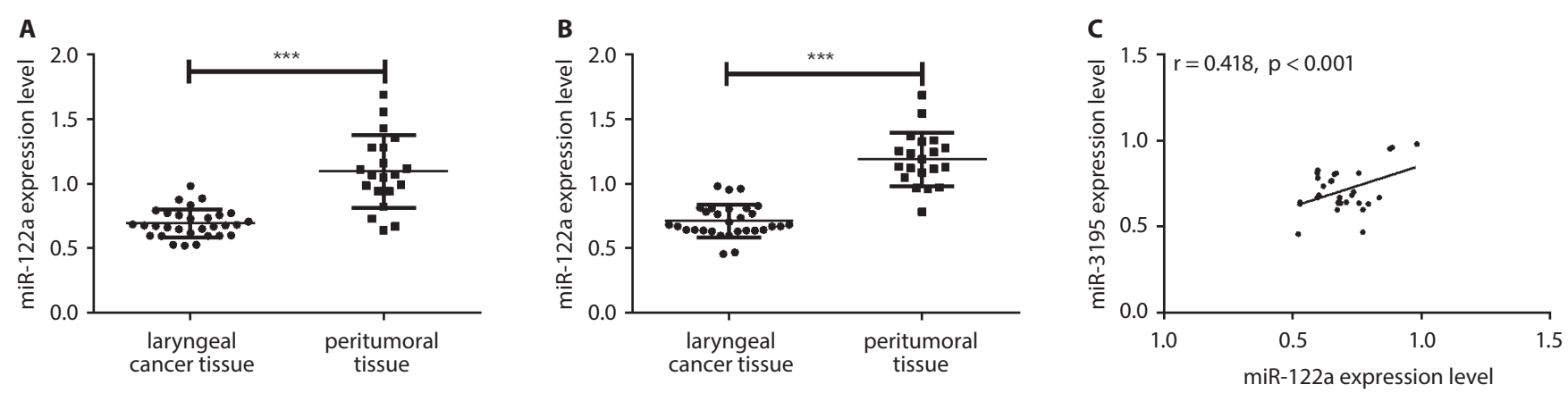

Fig. 1. miR-122a and miR-3195 expressions in the $L C$ and peritumoral tissue and their associations. A. miR-122a expressions in the $L C$ and peritumoral tissue. B. miR-3195 expressions in the LC and peritumoral tissue. C. Correlation between miR-122a and miR-3195 expression levels in the LC tissue 
Table 3. Associations of miR-122a and miR-3195 expressions with clinicopathological features (x \pm SD)

\begin{tabular}{|c|c|c|c|c|c|c|c|}
\hline Features & $\mathrm{n}$ & miR-122a & $\mathrm{F}$ & p-value & miR-3195 & $\mathrm{F}$ & $\mathrm{p}$-value \\
\hline Gender & & & 0.538 & 0.595 & & 0.329 & 0.744 \\
\hline male & 21 & $0.754 \pm 0.128$ & & & $0.826 \pm 0.193$ & & \\
\hline female & 9 & $0.726 \pm 0.137$ & & & $0.851 \pm 0.184$ & & \\
\hline Age & & & 1.585 & 0.124 & & 0.365 & 0.718 \\
\hline$\leq 62$ years & 16 & $0.767 \pm 0.136$ & & & $0.846 \pm 0.208$ & & \\
\hline$>62$ years & 14 & $0.691 \pm 0.125$ & & & $0.819 \pm 0.195$ & & \\
\hline Pathological grade & & & 0.167 & 0.847 & & 0.046 & 0.956 \\
\hline G1 & 11 & $0.732 \pm 0.109$ & & & $0.842 \pm 0.124$ & & \\
\hline G2 & 15 & $0.739 \pm 0.128$ & & & $0.834 \pm 0.153$ & & \\
\hline G3 & 4 & $0.701 \pm 0.083$ & & & $0.817 \pm 0.148$ & & \\
\hline Lymphatic metastasis & & & 1.036 & 0.309 & & 0.926 & 0.363 \\
\hline yes & 10 & $0.698 \pm 0.097$ & & & $0.794 \pm 0.113$ & & \\
\hline no & 20 & $0.746 \pm 0.129$ & & & $0.846 \pm 0.158$ & & \\
\hline Clinical stage & & & 4.678 & $<0.001$ & & 1.127 & 0.269 \\
\hline$|-| \mid$ & 12 & $0.926 \pm 0.157$ & & & $0.836 \pm 0.119$ & & \\
\hline III-IV & 18 & $0.702 \pm 0.106$ & & & $0.789 \pm 0.107$ & & \\
\hline
\end{tabular}

A
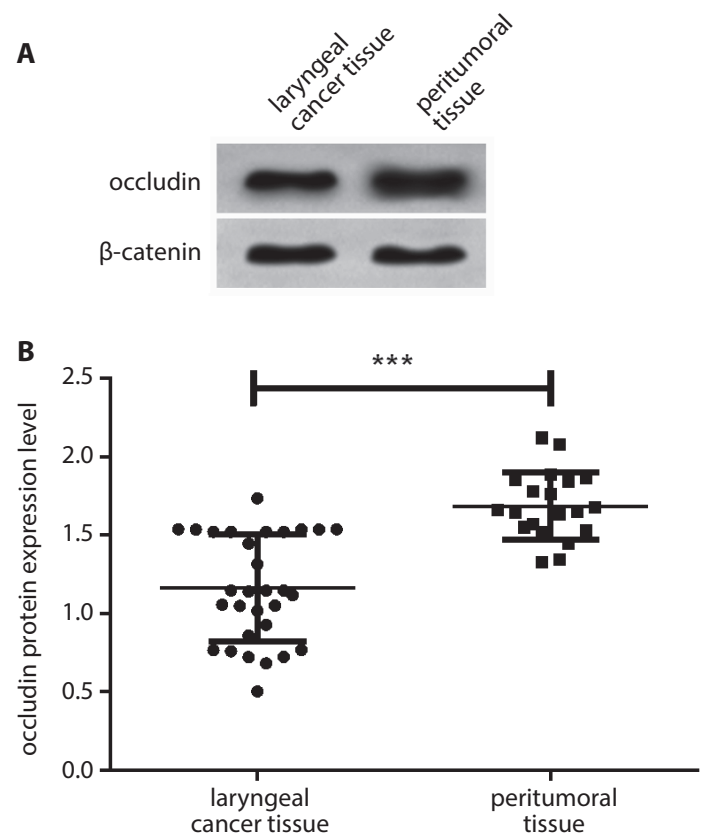

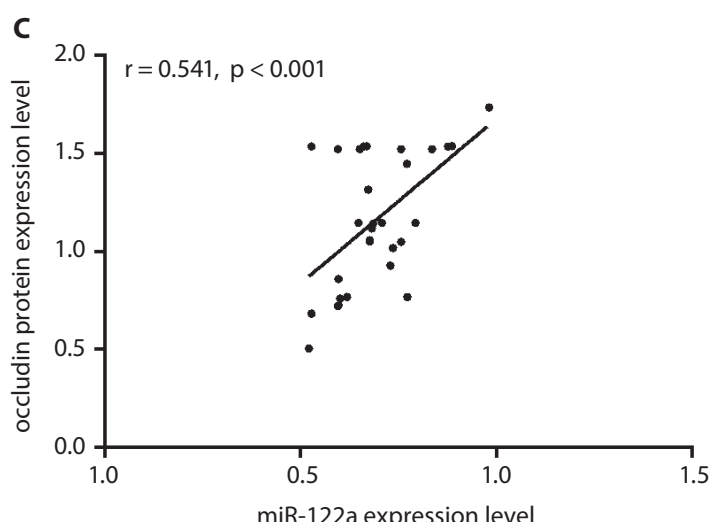

Fig. 2. Expression level of occludin protein in the LC tissue and its association with miR-122a. A. Protein band image. B. Occludin protein expression in LC and peritumoral tissue. C. Correlation between miR-122a expression level and occludin protein expression level in the LC tissue

LC - laryngeal cancer; ${ }^{* * *} \mathrm{p}<0.001$.

\section{miR-122a and miR-3195 expressions in Hep-2}

The qRT-PCR results showed that there were no differences in miR-122a and miR-3195 expression levels in Hep-2 between the non-transfected and the NG groups (both $\mathrm{p}>0.05$ ). The miR-122a expression level in the miR-122a-mimics group was greater than in the miR-122a-NG and the miR-122amimics-non-transfected groups, and the miR-3195 expression level in the miR-3195-mimics group was greater than in the miR-3195-NG and the miR-3195-mimics-nontransfected groups (all p $<0.001$; Fig. 4).

\section{Effect of miR-122a and miR-3195 overexpression on Hep-2 proliferation}

Effects of miR-122a and miR-3195 transfections after $24 \mathrm{~h}, 48 \mathrm{~h}, 72 \mathrm{~h}$, and $96 \mathrm{~h}$ on the proliferation of LC Hep-2 

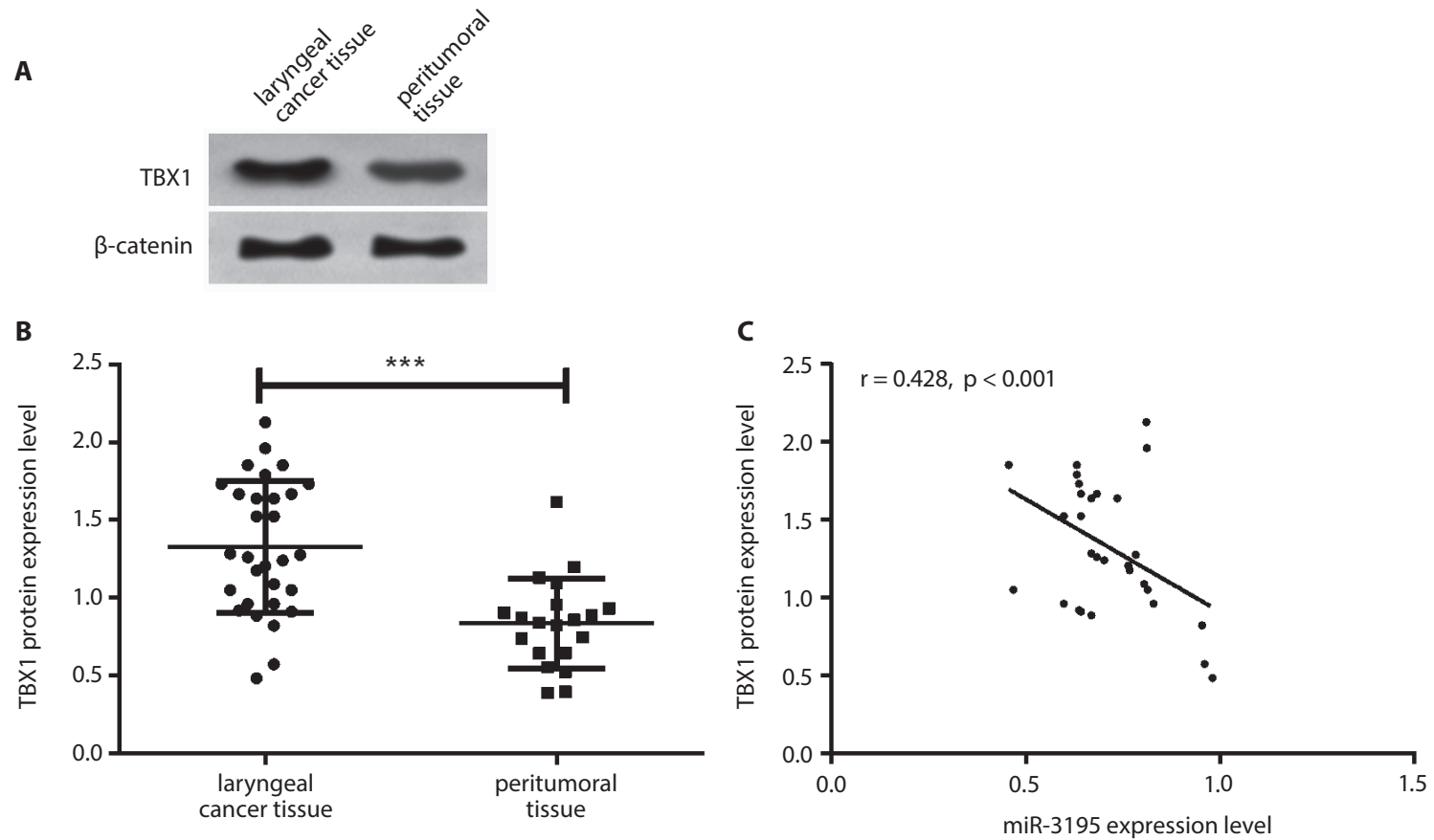

Fig. 3. Expression level of TBX1 protein in the LC tissue and its association with miR-3195. A. Protein band image. B. TBX1 protein expression in the LC and peritumoral tissue. C. Correlation between miR-3195 expression level and TBX1 protein expression level in the LC tissue

LC - laryngeal cancer; ${ }^{* * *} p<0.001$.
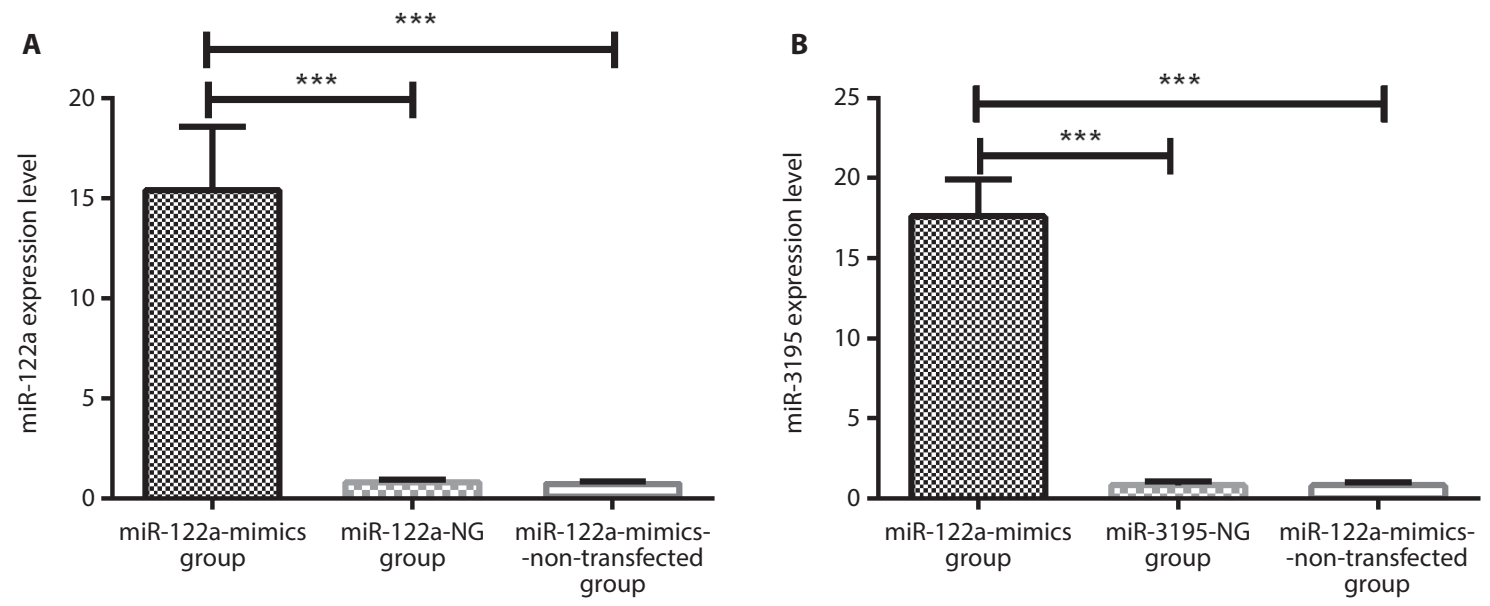

Fig. 4. miR-122a and miR-3195 expressions in LC Hep-2 cell. A. miR-122a expression in Hep-2. B miR-3195 expression in Hep-2

LC - laryngeal cancer; *** $p<0.001$

were investigated using MTT assay. The results showed that at different time points after $24 \mathrm{~h}$, the OD values in the miR-122a-mimics and the miR-3195-mimics groups were lower than in the miR-122a-NG and the miR-3195NG groups respectively (all $\mathrm{p}<0.001$; Fig. 5).

\section{Effect of miR-122a and miR-3195 overexpression on Hep-2 apoptosis}

Effects of miR-122a and miR-3195 transfections on the apoptosis of LC Hep-2 were studied. We used flow cytometry to measure the Hep-2 apoptosis rate after miR-122a and miR-3195 transfections. The results displayed that the cell apoptosis rate in the miR-122amimics group was higher than that in the miR-122a-NG and the miR-122a-mimics-non-transfected groups (both $\mathrm{p}<0.001$ ); and the apoptosis rate in the miR-3195-mimics group was higher than that in the miR-3195-NG and the miR-3195-mimics-non-transfected groups (both $\mathrm{p}<0.001$; Fig. 6).

\section{Effect of miR-122a overexpression on occluding expression in Hep-2 cell}

In order to explore the biological mechanisms of $\mathrm{miR}-122 \mathrm{a}$ in regulating Hep-2 cell proliferation 

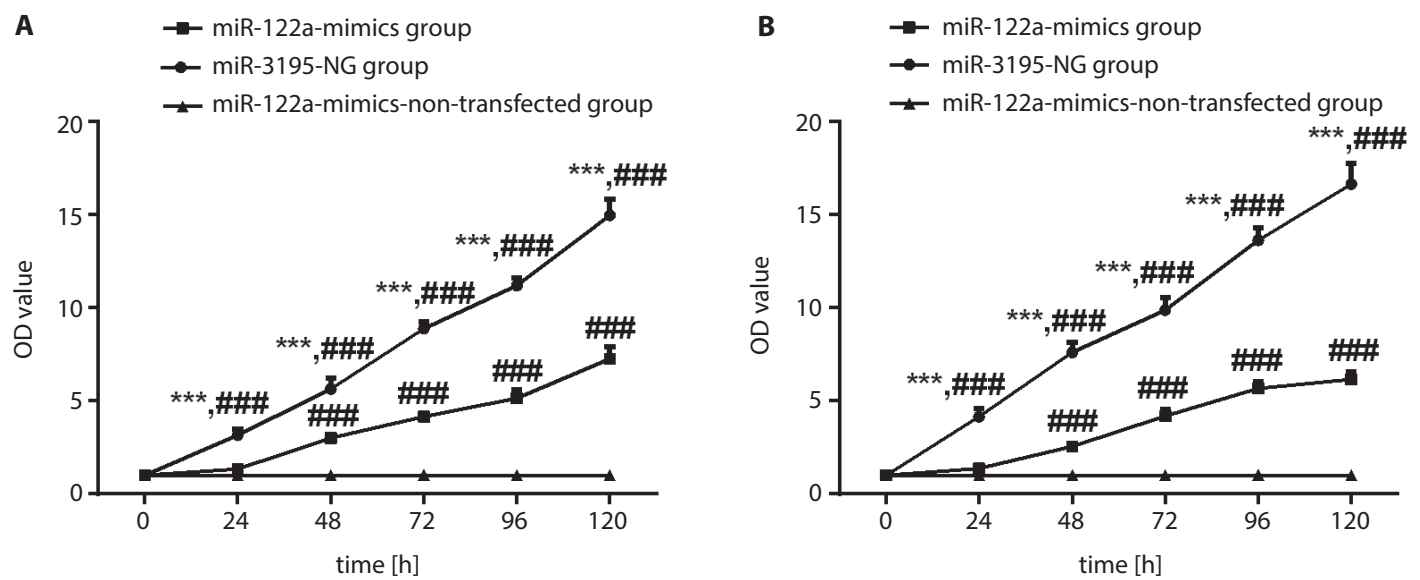

Fig. 5. Effect of miR-122a and miR-3195 overexpression on proliferation of LC Hep-2 cell. A. Effect of miR-122a overexpression on Hep-2 proliferation; ${ }^{* *} p<0.001$ vs the miR-122a-mimics group at the same time point; \#\#\# $p<0.001$ vs other time points within the same group. B. Effect of miR-3195 overexpression on HEP-2 cell proliferation; ${ }^{* *} p<0.001$ vs the miR-3195-mimics group at the same time point; $\# \# \#<0.001$ vs other time points within the same group

LC - laryngeal cancer.

and apoptosis, we performed western blot to measure the changes in occludin protein expression in Hep-2 following miR-122a overexpression. The results showed that, compared with the miR-122a-NG and the miR-122a-mimics-non-transfected groups, occludin protein expression level in the miR-122a-mimics group was higher ( $\mathrm{p}<0.001$; Fig. 7).

\section{Effect of miR-3195 overexpression on TBX1 expression in Hep-2 cell}

In order to investigate the biological mechanisms of miR-3195 in regulating Hep-2 cell proliferation and apoptosis, we performed western blot to measure the changes in TBX1 protein expression level in Hep-2 after miR-3195 overexpression. The results showed that, compared with the miR-3195-NG and the miR-3195-mimics-non-transfected groups, the TBX1 protein expression level in the miR-3195-mimics group was lower $(\mathrm{p}<0.001$; Fig. 8).

\section{Discussion}

The miRNA serves a critical role in the development of various cells and is closely related to cell differentiation, morphogenesis and tumor occurrence. ${ }^{11}$ The miRNA can either act as a tumor promoter or suppressor in the pathogenesis of different tumors. Since miRNA is closely associated with tumor occurrence and progression, miRNAs can become effective molecular biomarkers for the diagnosis, treatment and prognostic evaluation of tumor. ${ }^{12,13}$ As a liver-specific miRNA, miRNA-122a often acts as a tumor suppressor in cancer. In a study by Gramantieri et al., miR-122a was reported to be frequently downregulated in a cancerous human liver cell, and cyclin-G1 is its target protein. ${ }^{14}$ Zeisel et al. documented that miR-122 can regulate cholesterol metabolism and promote replication of hepatitis $C$ virus. Meanwhile, the reduction in miR-122 expression in hepatocellular carcinoma is associated with metastasis and poor prognosis. ${ }^{15}$ Lu et al., through analyzing miRNA genomic expression profile and bioinformatics target genes in LC, found a marked downregulation of miR-3195 level. ${ }^{16}$ Our study showed that miR-122a and miR-3195 expression levels in LC tissue were much lower than in peritumoral tissue, and the lowering of miR-122a was associated with the clinical stage of LC patients, which aligned with other study results. Both miR-122a and miR-3195 may act as tumor suppressors and be involved in the pathogenesis of LC. Moreover, we found that the expression levels of these 2 miRNAs in LC tissue were positively correlated with each other, suggesting a mutual regulation. However, there have been no studies in this area yet, and verification would be necessary in the future.

Much research has been carried out on the biological function of miR-122a in malignant tumors. Wang et al. have reported that miR-122a is downregulated in gastrointestinal cancer cell line and primary cancer tissue, while miR-122a mimics can inhibit cell growth. ${ }^{17}$ However, studies on the biological function of miR-3195 in tumor are scarce. In the present study, we analyzed the impact of miR-122a and miR-3915 transfections on the proliferation and apoptosis of Hep-2. The results showed that at different time points after $24 \mathrm{~h}$, the OD values in both miR-122a-mimics and miR-3195-mimics groups were much lower than in the NG groups, and the apoptosis rate in the 2 mimics groups was higher than in the NG and nontransfected groups. These findings indicated that overexpression of miR-122a and miR-3195 could markedly inhibit the proliferation of LC Hep-2 and promote its apoptosis.

Occludin is an integral membrane protein located at tight junction. Martin et al. observed that occludin could have an aberrant expression in cancerous human breast tissue and cancer cell line, and occludin expression loss is related 

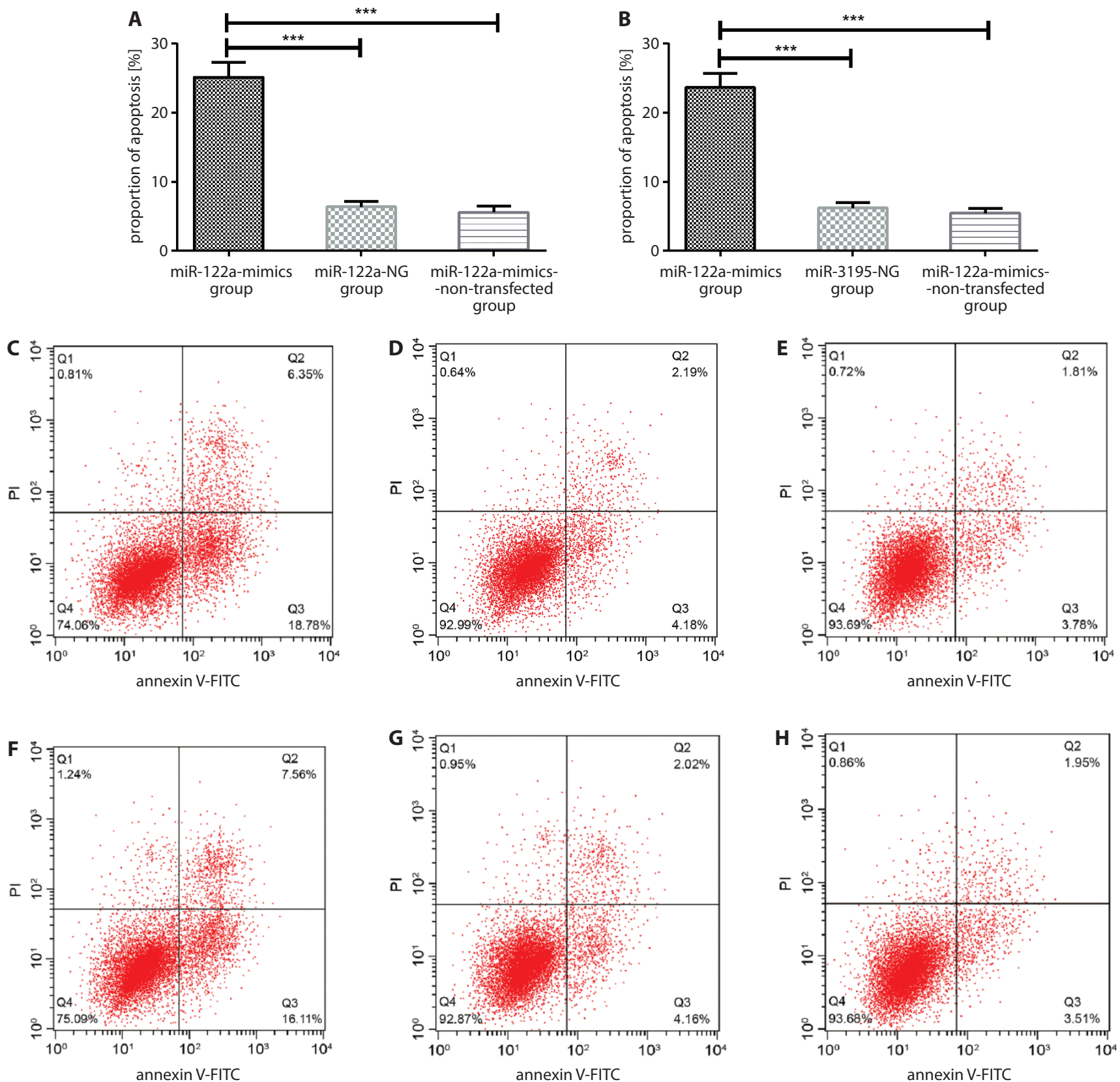

Fig. 6. Effect of miR-122a and miR-3195 overexpression on apoptosis of LC Hep-2 cell. A. Effect of miR-122a overexpression on Hep-2 apoptosis. B. Effect of miR-3195 overexpression on Hep-2 apoptosis. C. Hep-2 apoptosis rate in the miR-122a-mimics group. D. Hep-2 apoptosis rate in the miR-122a-mimics-NG group. E. Hep-2 apoptosis rate in the miR-122a-mimics-non-transfected group. F. Hep-2 apoptosis rate in the miR-3195-mimics group. G. Hep-2 apoptosis rate in the miR-3195-mimics-NG group. H. Hep-2 apoptosis rate in the miR-3195-mimics-non-transfected group

LC - laryngeal cancer; ${ }^{* * *} \mathrm{p}<0.001$.

to bone metastasis. ${ }^{18}$ In a study by Ye et al., it was reported that the binding of miR-122a to occludin 3'UTR can regulate occludin expression, and tumor necrosis factor $\alpha$ (TNF- $\alpha$ ) can adjust intestinal permeability through inducing miR-122a-mediated degradation of occludin, which substantiates the possibility of targeting miR-122a in vivo to maintain intestinal barrier function. ${ }^{19}$ Our results showed that the occludin protein expression level in LC tissue was lower compared with those in peritumoral tissues. Pearson analysis displayed that the miR-122a expression level was positively associated with the occludin protein level in LC tissue, suggesting that there may be a regulative relation between miR-122a and occludin. As a result, we performed western blot to measure the changes in occludin protein expression levels in Hep-2 after miR-122a overexpression. The results showed that, compared with the NG and non-transfected groups, the occludin protein level in the miR-122a-mimics group was higher. Therefore, miR-122a may inhibit Hep-2 cell proliferation and promote its apoptosis through upregulating occludin expression. Jingushi et al. have demonstrated that miR-122 can directly regulate occludin expression; moreover, their study 
A
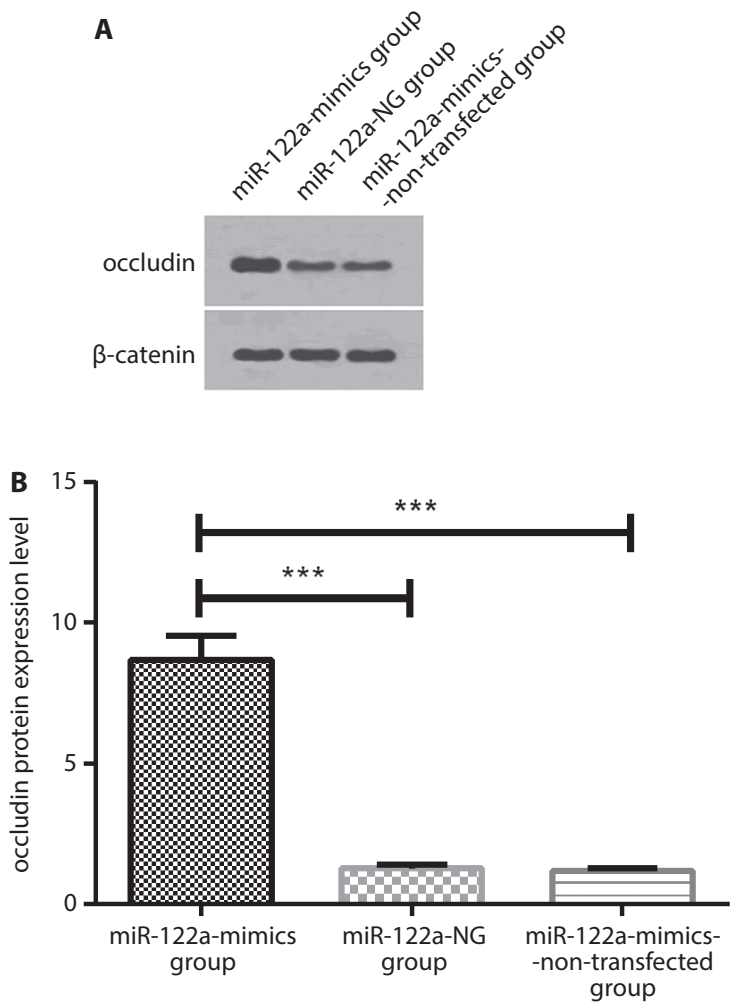

Fig. 7. Effect of miR-122a overexpression on occludin protein expression in LC Hep-2 cell. A. Protein band image. B. Occludin protein expression level in Hep-2 cell after miR-122a overexpression was detected using western blot

LC - laryngeal cancer; ${ }^{* * *} \mathrm{p}<0.001$.

also indicated that miR-122 level can be upregulated significantly in clear cell renal cell carcinoma (CCRCC), and the expression level of miR-122 is negatively associated with occludin protein expression level; the knockdown of occludin can promote CCRCC cell migration ability, but cannot enhance proliferation and invasion ability of CCRCC cell. ${ }^{20}$ This finding may be due to the difference in growth, invasion and regulatory mechanism between CCRCC and LC, and the mechanism is yet to be investigated.

TBX1 gene is mainly located on human chromosome $22 \mathrm{q}$ 11.21. The dysfunction of this gene can cause $22 \mathrm{q} 11$ deletion syndrome. $T B X 1$ can activate or inhibit transcription and negatively regulate cardiomyocyte differentiation. ${ }^{21}$ Aberrant expression of TBX1 can be found in various tumors. Jiang et al. have reported that TBX1 miRNA and protein are highly expressed in CCRCC and overexpression of TBX1 may contribute to CCRCC pathogenesis. ${ }^{22}$ Through measuring TBX1 protein expression in LC tissue, we found that TBX1 protein expression increased significantly in LC tissue, which was consistent with previous reports. Further studies demonstrated that miR-3195 was negatively associated with TBX1 protein expression in LC tissue. Thus, we speculate that there may be a regulative relation between miR-3195 and TBX1 protein. However, the correlation between them had not been elucidated in previous studies. Therefore, we predicted target genes
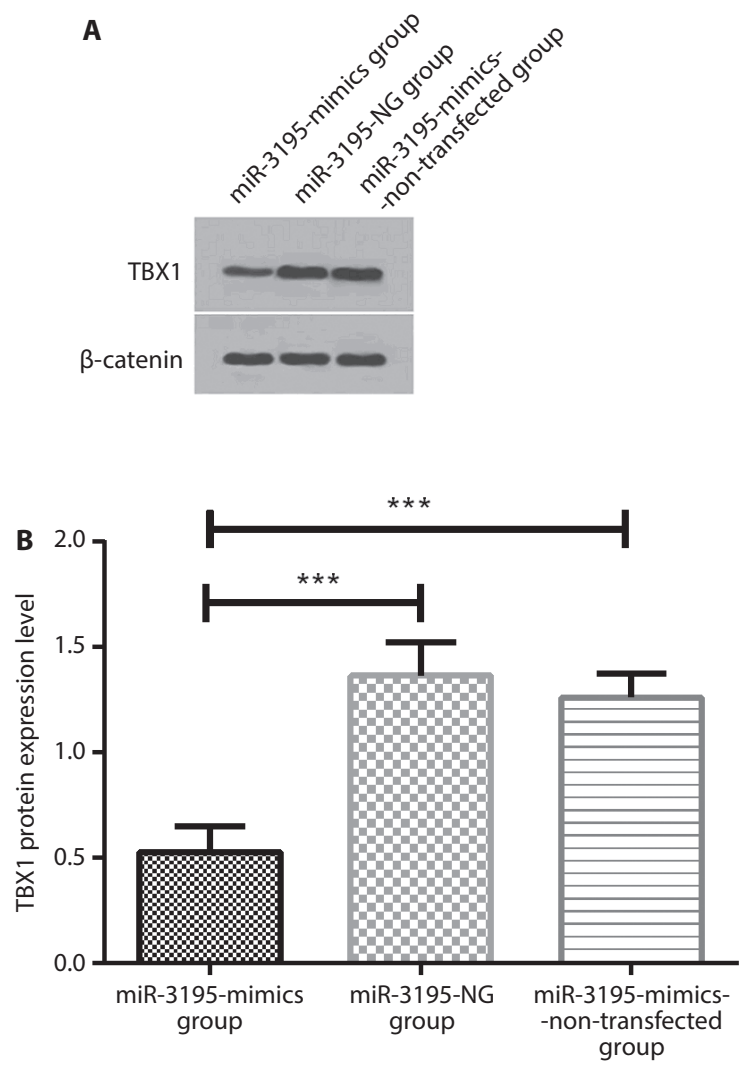

Fig. 8. Effect of miR-3195 overexpression on TBX1 protein expression in LC Hep-2 cell. A. Protein band image. B. TBX1 protein expression level in Hep-2 cell after miR-3195 overexpression was detected using western blot

LC - laryngeal cancer; ${ }^{* * *} \mathrm{p}<0.001$.

for miR-3195 and found TBX1 protein in the miR-3195 binding site. Western blot was used to detect changes in TBX1 protein expression in Hep-2 after miR-3195 overexpression. The results showed that, compared with the NG and non-transfected groups, TBX1 protein expression level was lower in the miR-3195-mimics group, indicating that miR-3195 may suppress Hep-2 cell proliferation and promote its apoptosis through inhibiting TBX1 expression. However, more studies would be necessary in the future to elucidate its detailed mechanism.

In the present study, we observed that miR-122a and miR-3195 overexpression could suppress Hep-2 cell proliferation and promote its apoptosis, and we carried out preliminary analysis on the mechanisms. However, there were still some limitations in the study design. First, we did not verify the relations between miR-122a and occludin, and between miR-3195 and TBX1 using dual-luciferase reporter assay. Second, the effects of miR-122a and miR-3195 on LC Hep-2 migration and invasion abilities were not further investigated, and the regulating mechanism remains unclear. Therefore, more studies would be required in the future to overcome these shortcomings and to verify our results.

In conclusion, both miR-122a and miR-3195 can act as tumor suppressors in LC. miR-122a is related to patients' clinical stage, and the 2 miRNAs may be involved in the pathogenesis of LC. Both miR-122a and miR-3195 can 
substantially inhibit Hep-2 cell proliferation and promote its apoptosis. These effects may be achieved by the upregulation of occludin expression by miR-122a and suppression of TBX1 expression by miR-3195. Therefore, miR-122a and miR-3195 may become potential targets for the treatment of LC.

\section{ORCID iDs}

Xingli Jiang (1) https://orcid.org/0000-0002-8584-926X

Zhiguang Gao (1) https://orcid.org/0000-0003-4025-7743

Linli Tian (1) https://orcid.org/0000-0001-8378-109X

Ming Liu (ㅇ) https://orcid.org/0000-0002-9366-2577

\section{References}

1. Tomeh C, Holsinger FC. Laryngeal cancer. Curr Opin Otolaryngol Head Neck Surg. 2014;22(2):147-153.

2. Miao S, Mao X, Zhao S, et al. miR-217 inhibits laryngeal cancer metastasis by repressing AEG-1 and PD-L1 expression. Oncotarget. 2017; 8(37):62143-62153.

3. Yu X, Li Z. The role of microRNAs expression in laryngeal cancer. Oncotarget. 2015;6(27):23297-23305.

4. Nie W, Ge HJ, Yang XQ, et al. LncRNA-UCA1 exerts oncogenic functions in non-small cell lung cancer by targeting miR-193a-3p. Cancer Lett. 2016;371(1):99-106.

5. Fernandez S, Risolino M, Mandia N, et al. miR-340 inhibits tumor cell proliferation and induces apoptosis by targeting multiple negative regulators of p27 in non-small cell lung cancer. Oncogene. 2015; 34(25):3240-3250.

6. Liu J, Tang Q, Li S, Yang X. Inhibition of HAX-1 by miR-125a reverses cisplatin resistance in laryngeal cancer stem cells. Oncotarget. 2016; 7(52):86446-86456.

7. Chen YH, Yu YF. Impact of miR-122a on inhibition proliferation of laryngeal carcinoma cell line Hep2 [in Chinese]. Chinese Journal of Immunology. 2017;33:352-355.

8. Yoon SO, Kim EK, Lee M, et al. NOVA1 inhibition by miR-146b-5p in the remnant tissue microenvironment defines occult residual disease after gastric cancer removal. Oncotarget. 2016;7(3):2475-2495.

9. Forastiere AA, Ismaila N, Wolf GT. Use of larynx-preservation strategies in the treatment of laryngeal cancer: American Society of Clinical Oncology Clinical Practice Guideline Update Summary. J Oncol Pract. 2018;14:123-128.
10. Munoz M, Rosso M, Aguilar FJ, Gonzalez-Moles MA, Redondo M, Esteban F. NK-1 receptor antagonists induce apoptosis and counteract substance $P$-related mitogenesis in human laryngeal cancer cell line HEp-2. Invest New Drugs. 2008;26(2):111-118.

11. Wan GL, Chen H, Zhou L, Huang JM. Overexpressed miR-128a inhibits the proliferation of laryngeal cancer cells. Trans/ Cancer Res. 2018;7(4): 901-911.

12. Wu T, Qu L, He G, et al. Regulation of laryngeal squamous cell cancer progression by the IncRNA H19/miR-148a-3p/DNMT1 axis. Oncotarget. 2016;7(10):11553-11566.

13. Jin C, Zhang Y, Li J. Upregulation of MiR-196a promotes cell proliferation by downregulating p27(kip1) in laryngeal cancer. Biol Res. 2016; 49(1):40.

14. Gramantieri L, Ferracin M, Fornari F, et al. Cyclin G1 is a target of miR-122a, a microRNA frequently downregulated in human hepatocellular carcinoma. Cancer Res. 2007;67(13):6092-6099.

15. Zeisel MB, Pfeffer S, Baumert TF. miR-122 acts as a tumor suppressor in hepatocarcinogenesis in vivo. J Hepatol. 2013;58(4):821-823.

16. Lu ZM, Lin YF, Jiang L, et al. Micro-ribonucleic acid expression profiling and bioinformatic target gene analyses in laryngeal carcinoma. Onco Targets Ther. 2014;7:525-533.

17. Wang $X$, Lam EK, Zhang J, Jin H, Sung JJ. MicroRNA-122a functions as a novel tumor suppressor downstream of adenomatous polyposis coli in gastrointestinal cancers. Biochem Biophys Res Commun. 2009;387(2):376-380.

18. Martin TA, Jordan N, Davies EL, Jiang WG. Metastasis to bone in human cancer is associated with loss of occludin expression. Anticancer Res. 2016;36(3):1287-1293.

19. Ye D, Guo S, Al-Sadi R, Ma TY. MicroRNA regulation of intestinal epithelial tight junction permeability. Gastroenterology. 2011;141(4): 1323-1333.

20. Jingushi K, Kashiwagi Y, Ueda $Y$, et al. High miR-122 expression promotes malignant phenotypes in $\mathrm{CCRCC}$ by targeting occludin. Int J Oncol. 2017;51(1):289-297.

21. Pane LS, Fulcoli FG, Cirino A, et al. Tbx1 represses Mef2c gene expression and is correlated with histone 3 deacetylation of the anterior heart field enhancer. Dis Model Mech. 2018;11(9). pii: dmm029967. doi:10.1242/dmm.029967

22. Jiang $H$, Tian $H$, Guo $Y$, et al. Expression of TBX1 gene in kidney tissues in patients with clear cell renal cell carcinoma and its clinical significance. Journal of China Medical University. 2016. 